\title{
Patient-reported pain, discomfort, and anxiety during magnetic resonance imaging- targeted prostate biopsy
}

Gregory T. Chesnut, MD'; Piotr Zareba, MD²; Daniel D. Sjoberg, MA ${ }^{3}$; Maha Mamoor, MS ${ }^{1}$; Sigrid Carlsson, MD, PhD, MPH ${ }^{1,3,4}$; Taehyoung Lee, $\mathrm{MD}^{1}$; Jonathan Fainberg, $\mathrm{MD}^{1}$; Emily Vertosick $^{3}$; Michael Manasia, BSN, RN ${ }^{1}$; Mary Schoen, MSN, MPH, NP-C ${ }^{1}$; Behfar Ehdaie, $\mathrm{MD}, \mathrm{MPH}^{1,3}$

${ }^{1}$ Department of Surgery, Urology Service, Memorial Sloan Kettering Cancer Center, New York, NY, United States; ${ }^{2}$ Department of Surgery, Urology Division, McMaster University, Hamilton, ON, Canada; ${ }^{3}$ Department of Epidemiology and Biostatistics, Memorial Sloan Kettering Cancer Center, New York, NY, United States; ${ }^{3}$ Institute of Clinical Sciences, Sahlgrenska Academy at Gothenburg University, Gothenburg, Sweden

Financial support: This work was supported in part by funds from the Sidney Kimmel Center for Prostate and Urologic Cancers, a Specialized Program of Research Excellence grant (P50-CA92629) from the National Cancer Institute to Dr. Howard Scher, a National Institutes of Health/National Cancer Institute Cancer Center Support Grant (P30-CA008748) to Memorial Sloan Kettering Cancer Center, and the David H. Koch prostate cancer research fund.

Cite as: Can Urol Assoc J 2019 November 29; Epub ahead of print. http://dx.doi.org/10.5489/cuaj.6102

Published online November 29, 2019

$* * *$

\section{Abstract}

Introduction: The addition of targeted prostate biopsy to systemic biopsy impacts patient experience. We examined patient-reported pain, discomfort, anxiety, and tolerability among men undergoing magnetic resonance imaging (MRI)-targeted prostate biopsy in addition to transrectal ultrasound-guided systematic biopsy compared to those undergoing systematic biopsy alone. Methods: All patients underwent transrectal systematic 14-core biopsies. Patients with regions of interest on MRI underwent additional targeted biopsies. All patients received equivalent periprostatic nerve block. Four single-item, standard 11-point numerical rating scales evaluating pain, discomfort, anxiety, and tolerability were completed immediately after biopsy. Differences in means were compared using t-tests. Correlation between rated domains was tested using Spearman's correlation coefficient. 
Results: Of 273 consecutive patients, 195 (71\%) underwent targeted biopsy and 188 (69\%) had undergone prior biopsy. In all men, the median score for pain and tolerability was 3 , while the median score for discomfort and anxiety was 4 . Pain was rated at 7 or above by $15 \%$ of patients. Moderate correlation between pain, discomfort, anxiety, and tolerability of repeat biopsy was observed (Spearman's $\rho$ between 0.48 and 0.76). Compared to patients undergoing systematic biopsy alone, men who received both targeted and systematic biopsies reported higher anxiety scores (difference 1.2; 95\% confidence interval [CI] 0.4-2.0; $p=0.004$ ) and discomfort (difference 1.0; 95\% CI 0.3-1.7; $\mathrm{p}<0.001$ ).

Conclusions: Patients undergoing targeted and systematic biopsies report more discomfort and anxiety than patients undergoing systematic biopsies alone. Absolute differences are small, and patients are willing to undergo repeat biopsy if advised. Interventions to reduce biopsy-related anxiety are needed.

\section{Introduction}

TRUS-guided prostate biopsy has long been considered the gold standard for diagnosing prostate cancer in men with an abnormal finding on DRE or an elevated serum PSA level. ${ }^{1,2}$ Although generally regarded as a well-tolerated procedure that can be safely performed in an outpatient setting, prostate biopsy is associated with significant morbidity in $<5 \%$ of men. ${ }^{3,4}$ In a contemporary prospective cohort of 1,147 British men undergoing prostate biopsy, approximately one-third experienced a moderate or major adverse event within 35 days after the procedure, with $1.4 \%$ requiring hospital admission. ${ }^{5}$ Even among men who do not experience complications, anxiety related to the experience of undergoing a biopsy is common and leads to significant biopsy-related distress in $49 \%$ of men,, 7 suggesting that the procedure itself also carries a significant psychological burden. Both the physical and psychological morbidity of serial prostate biopsies are barriers to active surveillance for men with low-risk prostate cancer.

Recently, MRI has transformed the diagnostic evaluation of suspected prostate cancer. MRI can identify tumors that may otherwise not be detected by a traditional systematic biopsy sampling scheme, especially in the anterior zone. ${ }^{8}$ Software that co-registers ultrasound and MRI has allowed effective targeting of these lesions during biopsy and increased the detection of higher-grade cancer compared to standard biopsy. ${ }^{9-11}$ Despite the increased detection rates seen on MRI-targeted prostate biopsies, the combination of targeted biopsy with systematic biopsy still offers the best detection rate for prostate cancer and many biopsy and surveillance protocols rely on both these types of biopsies. ${ }^{12}$

The few potential drawbacks of adding targeted biopsy to systematic biopsy in an outpatient setting include an increase in pain related to the greater number of cores collected and longer procedural times. Furthermore, patients may experience greater anxiety due to the 
knowledge that there are imaged areas suspicious for cancer. While many studies have characterized pain, discomfort, and anxiety related to systematic prostate biopsy, the patient experience among men undergoing targeted biopsy in addition to systematic biopsy has not been adequately studied. We compared the experience of men undergoing MRI-targeted and TRUSguided systematic biopsies with the experience of those undergoing TRUS-guided systematic biopsy alone at our institution. Secondary objectives were to determine how patient-reported anxiety regarding biopsy impacted pain and attitudes toward repeat biopsy and to compare the experience of patients undergoing first versus repeat biopsy procedures.

\section{Methods}

The study was approved by Memorial Sloan Kettering Cancer Center's Institutional Review Board. A short, 4-question quality-of-life tool was created to capture patients' experience of undergoing prostate biopsy. Patients were asked the following questions related to their biopsy: "Overall, how much pain did you experience during the biopsy?" (referred to as the Pain Scale); "Overall, how much discomfort did you experience during the biopsy?" (referred to as the Discomfort Scale); "Overall, how anxious or tense were you during the biopsy?" (referred to as the Anxiety Scale); and, "Overall, how would you feel about having to repeat a biopsy?" (referred to as the Tolerability Scale). Each patient rated their answer on a standard numerical rating scale from 0 to 10 , with 0 representing no pain, discomfort, or anxiety, and 10 representing very severe pain, discomfort, or anxiety. ${ }^{13}$ For the Tolerability Scale, a score of 0 corresponded to the patient being able to tolerate a repeat biopsy with "no problem" and 10 to the patient being unable to tolerate a repeat biopsy. These questionnaires were prospectively provided to 273 consecutive patients receiving prostate biopsy at our institution between April and October of 2015. All biopsies were performed by a single urologist (BE) and informed consent was obtained. The questionnaire was integrated into post-procedural care and completed immediately after completion of the biopsy by all patients during the study period. The patients were consecutively enrolled, representing the mix of men undergoing biopsy at our institution at the time. All men received identical periprocedural care. Men were not randomized to either systematic plus targeted biopsy or systematic biopsy alone but were pragmatically evaluated based on the type of biopsy performed. Biopsy technique was chosen based on imaging and clinical indications prior to enrollment into the study. All men underwent pre-biopsy MRI and all men who had targetable lesions underwent targeted biopsy in addition to systematic biopsy.

All patients underwent a transrectal systematic 14-core prostate biopsy using TRUS guidance, taking cores from medial and lateral base, mid-prostate, and apex as well as one from the transition zone on the left and right side. Patients in whom an abnormal lesion was identified on MRI also underwent two additional biopsies targeting the lesion. Typically, one targeted core was obtained with cognitive visual targeting and another attained using MRI-ultrasound fusion software (UroStation, Koelis, Grenoble, France), with optimum targeting technique based on 
surgeon preference to optimize detection of prostate cancer in the region of interest. ${ }^{14}$ All biopsies were collected using an 18-gauge Tru-Cut needle. Indications for biopsy included elevated serum PSA levels, active surveillance in patients with prostate cancer, or suspicious findings on MRI or DRE. Analgesia was administered prior to the biopsy in the form of a PNB using $2 \%$ plain lidocaine; $5 \mathrm{~mL}$ was injected on either side of the prostate lateral to the neurovascular bundle. ${ }^{15}$ No patients received peri-procedural sedation or anxiolytics.

Statistics

We used t-tests to assess the significance of differences in mean pain, discomfort, and anxiety scores between patients who received systematic biopsy only and patients who received both systematic and targeted biopsy. To assess the monotonic relationship between each pair of scales, Spearman's correlation coefficient was applied. Locally weighted polynomial regression was used to examine relationships among the scales.

\section{Results}

Patient characteristics are summarized in Table 1. Of the 273 patients referred for prostate biopsy, 195 (71\%) underwent systematic and targeted biopsy, and 188 (69\%) had had a prior prostate biopsy. The most common indication for biopsy was active surveillance in men with prostate cancer (40\%), followed by elevated serum PSA $(33 \%)$.

Figure 1 shows the score distribution of the 4 outcomes. The median score for the pain and tolerability scales was 3, and the median score for the discomfort and anxiety scales was 4 . Pain levels of 7 or above were reported by $15 \%$ of patients, and anxiety levels of 7 or above by $24 \%$ of patients. Across all scales, $41 \%$ of patients reported a score of 7 or higher on at least one domain.

Patients who received both systematic and targeted biopsies were more anxious (difference of $1.2 ; 95 \%$ CI $0.4,2.0 ; p=0.004$ ) and experienced more discomfort (difference of $1.0 ; 95 \%$ CI $0.3,1.7 ; p=0.005$ ) than those who received systematic biopsy only (Table 2 ). Patients undergoing systematic and targeted biopsies also reported higher pain levels, but the differences were not statistically significant (difference $0.6 ; 95 \%$ CI $0.0-1.2 ; p=0.067$ ). We did not observe any significant differences in pain, discomfort, anxiety, or tolerability between patients who had or had not undergone a prior prostate biopsy (Table 3 ).

All 4 scales were significantly correlated with one another (fig. 2). However, the levels of association were moderate, with Spearman correlation coefficients ranging from 0.48 to 0.76 . The pain and tolerability scales showed the lowest correlation of any pair (Spearman's $\rho=0.48$ ), and pain and discomfort exhibited the highest correlation $(\rho=0.76)$. All other correlations ranged between 0.53 and 0.60 . Due to the modest pairwise correlations, excluding any one of the scales would substantively reduce information about a patient. 


\section{Discussion}

MRI-targeted biopsy is increasingly used in prostate biopsies, particularly in the active surveillance population. ${ }^{16}$ Biopsy techniques that combine targeted biopsy with systematic biopsy have been shown to increase the detection of clinically significant prostate cancer. Compared with targeted biopsy alone, the addition of systematic biopsy to targeted biopsy avoids the risk of missing up to $15 \%$ of significant cancers. ${ }^{17}$ As a moderate proportion of men undergoing active surveillance experience disease-related anxiety, ${ }^{18}$ evaluating the impact of anxiety on biopsy can aid in surveillance-related counseling and may improve active surveillance compliance. We sought to compare biopsy-related pain, discomfort, and anxiety between patients undergoing systematic biopsy alone with patients undergoing both systematic and MRI-targeted biopsy.

Uniquely, our study evaluated the different impact biopsy technique had on these four patient-reported domains. The standard 11-point NRS is established and validated in evaluation of pain and discomfort. ${ }^{19-21}$ Similarly, the use of 11-point NRS has been used to evaluate perioperative anxiety and was shown to correlate with physiologic stress response in preoperative patients. ${ }^{22,23} \mathrm{NRS}$ has also been used to evaluate willingness to undergo repeat procedures. ${ }^{24}$ Our study is unique in combining all four domains in NRS evaluations immediately after prostate biopsy.

Notably, our data demonstrates that MRI-targeted biopsy is associated with increases in both patient discomfort and anxiety, and that these domains are moderately correlated to each other with a Spearman $\rho=0.60$. Though patients undergoing both MRI-targeted and systematic biopsies reported more discomfort, their tolerability of future biopsy did not differ from that of patients undergoing systematic biopsy alone. While pain was increased among the targeted biopsy with systemic biopsy cohort, the increase was smaller and not statistically significant. Importantly, we found that reporting of pain, anxiety, discomfort, and tolerability were separately and uniquely correlated; therefore, a survey of each item is important to understand the experience of patients during prostate biopsy.

TRUS-guided systematic prostate biopsy has been the established method of prostate biopsy detection for the past 3 decades. The procedure causes significant pain and discomfort, as evidenced by the extensive body of research, including over 100 randomized trials, into interventions aimed at decreasing biopsy-related pain. ${ }^{25}$ The most effective of these, the PNB, renders prostate biopsy tolerable in most patients; studies employing PNB fairly consistently report mean pain scores between 2 and 4 on 11-point NRS. ${ }^{26-30}$ Congruent with these findings, median pain, discomfort, and anxiety scores in our series of 273 patients were 3,4 , and 4 , respectively. Nevertheless, $41 \%$ of men in our series reported severe $(>7 / 10)$ pain, discomfort and/or anxiety, suggesting that prostate biopsy remains an uncomfortable and anxiety-inducing procedure despite PNB in a substantial proportion of men. Fifteen percent of men described severe $(>7 / 10)$ pain associated with the procedure. 
This is a novel finding that has been obscured in past studies by almost exclusive reporting of average pain scores and not the proportion of patients who report significant pain or discomfort. It also suggests the need to identify those men at highest risk of experiencing significant pain, such as those with high pre-biopsy anxiety, ${ }^{31-33}$ so that they may receive additional interventions. Prior biopsy or active surveillance status was not associated with a score of $\geq 7$ on the measured domains of pain, discomfort, anxiety, or tolerance of procedure, indicating that further research is warranted to prospectively identify men at greatest risk for biopsy-related pain, anxiety, and discomfort.

Importantly, we found only moderate correlation between measures of pain, discomfort, anxiety, and tolerability, defined as the attitude toward repeat biopsy. Prior prostate biopsy series have reported tolerability rates exceeding $80 \% .{ }^{34-36}$ However, only three studies have assessed the correlation between the biopsy experience and willingness to undergo repeat biopsy. Robins et al. showed no difference in patient-reported pain or discomfort when comparing systematic biopsy and targeted biopsy with systematic biopsy alone and that both groups were willing to repeat the biopsy, if advised. ${ }^{24}$ In a study of 476 Australian men undergoing prostate biopsy with PNB, only $12(2.5 \%)$ said that they would be unable to tolerate a repeat biopsy without sedation or a general anesthetic, and these 12 men reported significantly higher pain scores during probe insertion, $\mathrm{PNB}$, and biopsy. ${ }^{36} \mathrm{We}$ attribute the moderate correlation between pain and discomfort and tolerability to the significant proportion of men in this study who had undergone prior biopsy and were thus prepared for the experience. This complements the findings of Carlsson et al. that surveillance-biopsy related anxiety significantly decreased over time within the Gothenburg branch of the European Randomized Study of Screening for Prostate Cancer. ${ }^{37}$ Based on our findings, we hypothesize that counseling biopsy-naïve men on the experience of prostate biopsy and setting expectations using appropriate framing techniques will reduce anxiety and improve tolerability.

Although we report significantly greater discomfort and anxiety among men undergoing targeted and systematic biopsies compared with those undergoing systematic biopsy alone, the mean absolute difference was approximately 1 point on an 11-point NRS. The likelihood that this increase in discomfort and anxiety is clinically significant seems low, as it did not decrease tolerability of future repeat prostate biopsy. The minimal clinically important difference in pain scores as measured on an 11-point NRS has been variably defined in the anesthesia literature as either $1.3^{38,39}$ or 2 points, ${ }^{40,41}$ and it would seem reasonable to extrapolate this minimal clinically important difference to our measures of pain and discomfort. Thus, the additional discomfort and anxiety conferred by adding targeted biopsy to a systematic biopsy scheme does not appear to outweigh the benefits of this approach, namely enhanced sensitivity of cancer detection.

The observed increases in discomfort and anxiety scores with the addition of targeted biopsy could be explained by the larger number of cores sampled and longer procedural duration. 
On average, 16 cores were sampled during targeted and systematic biopsy, compared to 14 cores during systematic biopsy only. As prior studies have shown that increasing the number of cores does not significantly increase pain or discomfort even in the absence of $\mathrm{PNB},{ }^{42,43}$ this is unlikely to be the main contributing factor. An alternative explanation is that because targeted biopsies were performed only in patients who had a lesion documented on MRI, they may have thought of themselves as being more likely to have a positive biopsy, increasing their anxiety. It is likely that a combination of adding the additional targeted biopsy cores to the procedure and the patient's knowledge that a targetable lesion exists combine to increase the patient's experience of discomfort and anxiety at prostate biopsy. This suggests that techniques which manage patient expectations when evaluating a targetable lesion may offer the potential of improved patient experience at time of targeted biopsy.

Strengths of our study include measurement of multiple domains of the patient experience using a simple numerical rating scale that has been applied extensively in prior studies of prostate biopsy, ${ }^{44}$ as well as having the questionnaire filled out by patients directly and confidentially, thus reducing bias. All evaluations were performed after biopsy by a single urologist experienced with image-guided prostate biopsy techniques, thus limiting variation in patient-provider counseling and overall biopsy experience. Though a single surgeon's bedside manner may not be generalizable to all settings, it can serve as a benchmark to evaluate patientreported pain, discomfort, anxiety, and tolerability in future studies and ongoing evaluations of patient experiences. Another potential limitation is that we assessed tolerability immediately after biopsy, while prior studies have shown that willingness to undergo repeat biopsy may be affected by biopsy-related symptoms that occur days and weeks after the procedure. ${ }^{5,45}$ The generalizability of our findings may also be limited by the relatively small number of cores collected during MRI-targeted biopsy ( 2 is our standard practice); this procedure could add significantly more pain and discomfort at other institutions. As our study included all consecutive men undergoing prostate biopsy for any reason at our institution, we did not randomize men to receive targeted plus systematic biopsy versus systematic biopsy alone. As such, there may be differences in these cohorts that disproportionally impact patient-reported outcomes. Further evaluations within these groups are topics of ongoing research at our institution.

\section{Conclusions}

Men undergoing targeted and systematic prostate biopsies experience more discomfort and anxiety during the procedure than those undergoing systematic biopsy alone. However, the absolute difference is small and therefore its clinical significance is uncertain. As MRI-targeted biopsies become more widely adopted, interventions aimed at decreasing patient biopsy-related anxiety will become more important; this may result in less procedural discomfort and improved active surveillance compliance. 


\section{References}

1. Hodge KK, McNeal JE, Stamey TA. Ultrasound guided transrectal core biopsies of the palpably abnormal prostate. J Urol 1989; 142: 66-70.

2. Hodge KK, McNeal JE, Terris MK, et al. Random systematic versus directed ultrasound guided transrectal core biopsies of the prostate. J Urol 1989; 142: 71-74.

3. Loeb S, Carter HB, Berndt SI, et al. Complications after prostate biopsy: data from SEER-Medicare. J Urol 2011; 186: 1830-34.

4. Loeb S, van den Heuvel S, Zhu X, et al. Infectious complications and hospital admissions after prostate biopsy in a European randomized trial. Eur Urol 2012; 61: 1110-14.

5. Rosario DJ, Lane JA, Metcalfe C, et al. Short term outcomes of prostate biopsy in men tested for cancer by prostate specific antigen: prospective evaluation within ProtecT study. BMJ 2012; 344: d7894.

6. Macefield RC, Metcalfe C, Lane JA, et al. Impact of prostate cancer testing: an evaluation of the emotional consequences of a negative biopsy result. Br J Cancer 2010; 102: $1335-40$.

7. Sharp L, Morgan E, Drummond FJ, et al. The pshycological impact of prostate biopsy: Prevalence and predictors of procedure-related distress. Psychooncology 2018;27(2):500-7.

8. Futterer JJ, Briganti A, De Visschere P, et al. Can clinically significant prostate cancer be detected with multiparametric magnetic resonance imaging? A systematic review of the literature. Eur Urol 2015; 68: 1045-53.

9. Moore CM, Robertson NL, Arsanious N, et al. Image-guided prostate biopsy using magnetic resonance imaging-derived targets: a systematic review. Eur Urol 2013; 63: 125-40.

10. Schoots IG, Roobol MJ, Nieboer D, et al. Magnetic resonance imaging-targeted biopsy may enhance the diagnostic accuracy of significant prostate cancer detection compared to standard transrectal ultrasound-guided biopsy: a systematic review and meta-analysis. Eur Urol 2015; 68: 438-50.

11. Siddiqui MM, Rais-Bahrami S, Turkbey B, et al. Comparison of MR/ultrasound fusionguided biopsy with ultrasound-guided biopsy for the diagnosis of prostate cancer. JAMA 2015; 313: 390-97.

12. Fourcade A, Payrard C, Tissot V, et al. The combination of targeted and systematic prostate biopsies is the best protocol for the detection of clinically significant prostate cancer. Scand J Urol 2018; 52: 174-79.

13. Langley GB, Sheppeard H. The visual analogue scale: its use in pain measurement. Rheumatol Int 1985; 5: 145-48.

14. Lee DJ, Recabal P, Sjoberg DD, et al. Comparative effectiveness of targeted prostate biopsy using magnetic resonance imaging ultrasound fusion software and visual targeting: a prospective study. J Urol 2016; 196(3): 697-702. 
15. Li M, Wang Z, Li H, et al: Local anesthesia for transrectal ultrasound-guided biopsy of the prostate: A meta-analysis. Sci Rep 2017; 7: 40421.

16. Sarkar D. The role of multi-parametric MRI and fusion biopsy for the diagnosis of prostate cancer - a systematic review of current literature. Adv Exp Med Biol 2018; 1095: 111-23.

17. Ploussard G, Borgmann H, Briganti A, et al. Positive pre-biopsy MRI: are systematic biopsies still useful in addition to targeted biopsies? World J Urol 2019; 37(2): 243-51.

18. Marzouk K, Assel M, Ehdaie B, et al. Long-term cancer specific anxiety in men undergoing active surveillance of prostate cancer: Findings from a large prospective cohort. J Urol 2018;200(6):1250-55.

19. Safikhani S, Gries KS, Trudeau JJ, et al. Response scale selection in adult pain measures: results from a literature review. J Patient Rep Outcomes 2017; 2: 40.

20. Petti E, Scher C, Meador L, et al. Can multidimensional pain assessment tools help improve pain outcomes in the perianesthesia setting? J Perianesth Nurs 2018; 33: 76772.

21. Dworkin RH, Turk DC, Farrar JT, et al. Core outcome measures for chronic pain clinical trials: IMMPACT recommendations. Pain 2005; 113: 9-19.

22. Hicks JA, Jenkins JG. The measurement of preoperative anxiety. J R Soc Med 1988; 81(9): 517-19.

23. Fell D, Derbyshire DR, Maile CJ, et al. Measurement of plasma catecholamine concentrations. An assessment of anxiety. Br J Anaesth 1985; 57: 770-74.

24. Robins D, Lipsky M, RoyChoudry A, et al. Assessment of discomfort and pain in patients undergoing fusion magnetic resonance imaging-guided vs TRUS-guided prostate biopsy. Urology 2018; 116: 30-34.

25. Lee C, Woo HH. Current methods of analgesia for transrectal ultrasonography (TRUS)guided prostate biopsy -- a systematic review. BJU Int 2014; 113 Suppl 2: 48-56.

26. Leibovici D, Zisman A, Siegel YI, et al. Local anesthesia for prostate biopsy by periprostatic lidocaine injection: a double-blind placebo controlled study. J Urol 2002; 167: 563-65.

27. Moussa AS, El-Shafei A, Diaz E, et al. Identification of the variables associated with pain during transrectal ultrasonography-guided prostate biopsy in the era of periprostatic nerve block: the role of transrectal probe configuration. BJU Int 2013; 111: 1281-86.

28. Nambirajan T, Woolsey S, Mahendra V, et al. Efficacy and safety peri-prostatic local anaesthetic injection in trans-rectal biopsy of the prostate: a prospective randomised study. Surgeon 2004; 2: 221-24.

29. Song SH, Kim JK, Song K, et al. Effectiveness of local anaesthesia techniques in patients undergoing transrectal ultrasound-guided prostate biopsy: a prospective randomized study. Int J Urol 2006; 13: 707-10.

30. Stirling BN, Shockley KF, Carothers GG, et al. Pain perception during transrectal ultrasound guided prostate needle biopsy: an objective analysis of local anesthesia use. Prostate Cancer Prostatic Dis 2002; 5: 209-11. 
31. Saracoglu T, Unsal A, Taskin F, et al. The impact of pre-procedural waiting period and anxiety level on pain perception in patients undergoing transrectal ultrasound-guided prostate biopsy. Diagn Interv Radiol 2012; 18: 195-99.

32. Tekdogan U, Tuncel A, Nalcacioglu V, et al. Is the pain level of patients affected by anxiety during transrectal prostate needle biopsy? Scand J Urol Nephrol 2008; 42: 2428.

33. Zisman A, Leibovici D, Kleinmann J, et al. The impact of prostate biopsy on patient well-being: a prospective study of pain, anxiety and erectile dysfunction. J Urol 2001; 165: 445-54.

34. Kahriman G, Donmez H, Mavili E, et al. Transrectal ultrasound guided multi-core prostate biopsy: pain control: results of 106 patients. J Clin Ultrasound 2011; 39: 27073.

35. Mkinen T, Auvinen A, Hakama M, et al. Acceptability and complications of prostate biopsy in population-based PSA screening versus routine clinical practice: a prospective, controlled study. Urology 2002; 60: 846-50.

36. Hossack T, Woo HH. Acceptance of repeat transrectal ultrasonography guided prostate biopsies with local anaesthesia. BJU Int 2011; 107 Suppl 3: 38-42.

37. Carlsson S, Aus G, Wessman C, et al. Anxiety associated with prostate cancer screening with special reference to men with a positive screening test (elevated PSA) - Results from a prospective, population-based, randomised study. Eur J Cancer 2007; 43: 210916.

38. Cepeda MS, Africano JM, Polo R, et al. What decline in pain intensity is meaningful to patients with acute pain? Pain 2003; 105: 151-57.

39. Gallagher EJ, Liebman M, Bijur PE. Prospective validation of clinically important changes in pain severity measured on a visual analog scale. Ann Emerg Med 2001; 38: 633-38.

40. Farrar JT, Berlin JA, Strom BL. Clinically important changes in acute pain outcome measures: a validation study. J Pain Symptom Manage 2003; 25: 406-11.

41. Farrar JT, Portenoy RK, Berlin JA, et al. Defining the clinically important difference in pain outcome measures. Pain 2000; 88: 287-94.

42. Chang SS, Alberts G, Wells N, et al. Intrarectal lidocaine during transrectal prostate biopsy: results of a prospective double-blind randomized trial. J Urol 2001; 166: 217880.

43. Naughton CK, Ornstein DK, Smith DS, et al.. Pain and morbidity of transrectal ultrasound guided prostate biopsy: a prospective randomized trial of 6 versus 12 cores. $J$ Urol 2000; 163: 168-71.

44. Hergan L, Kashefi C, Parsons JK. Local anesthetic reduces pain associated with transrectal ultrasound-guided prostate biopsy: a meta-analysis. Urology 2007; 69: 52025.

45. Welch HG, Fisher ES, Gottlieb DJ, et al. Detection of prostate cancer via biopsy in the Medicare-SEER population during the PSA era. J Natl Cancer Inst 2007; 99: 13951400 . 


\section{Figures and Tables}

Fig. 1. Distributions of quality of life (QoL) scales. For each scale, patients rated their experience during biopsy, except for the tolerability scale, which refers to the patients' willingness to undergo a repeat biopsy.
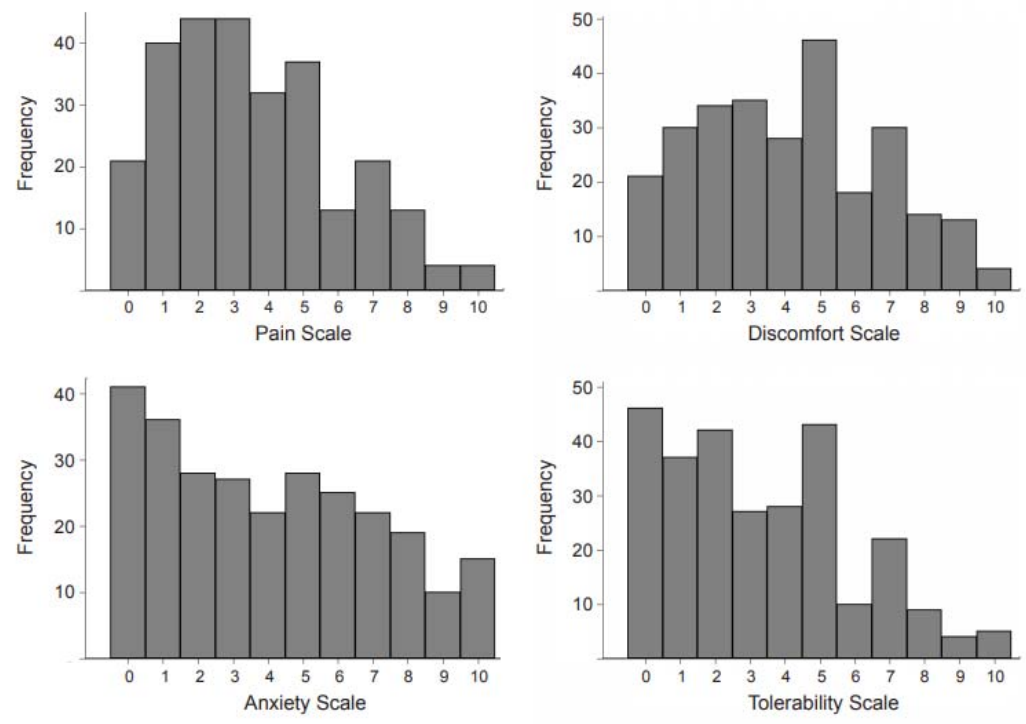

Fig. 2. Relationships among the quality of life (QoL) scales.
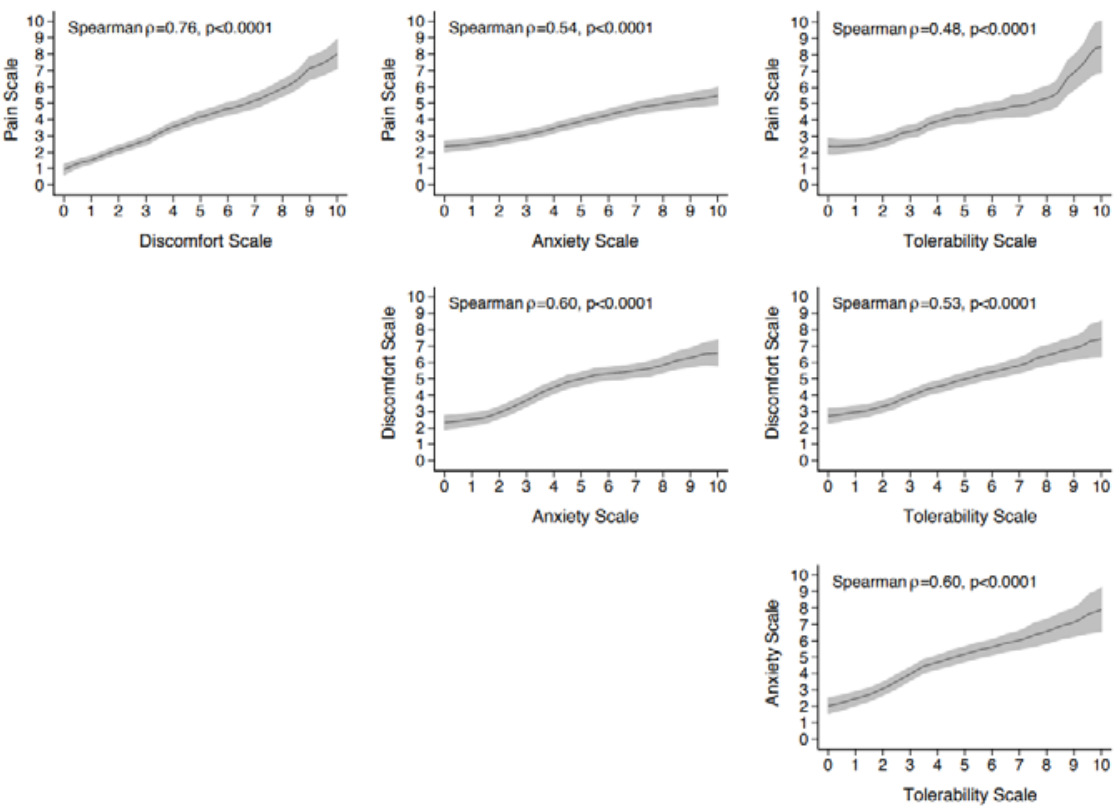


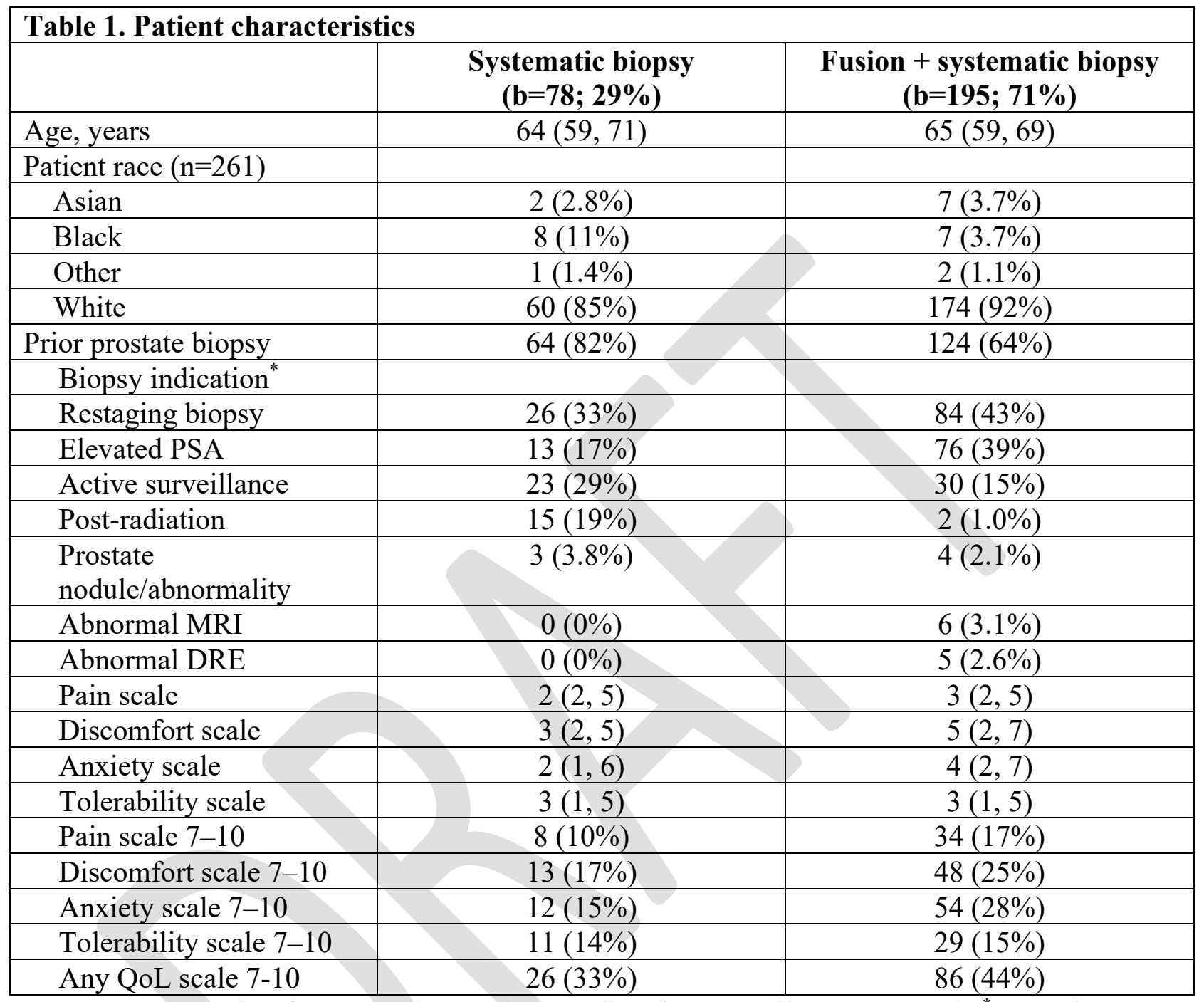

Data are presented as frequency (percent) or median (interquartile range [IQR]). ${ }^{*}$ More than one biopsy indication may apply to each patient. DRE: digital rectal examination; MRI: magnetic resonance imaging; PSA: prostate specific antigen; QoL: quality of life. 


\begin{tabular}{|l|c|c|c|c|c|}
\hline $\begin{array}{l}\text { Table 2. Differences in quality of life scales by biopsy scheme (targeted and systematic } \\
\text { vs. systematic only) }\end{array}$ & $\begin{array}{c}\text { Systematic } \\
\text { biopsy } \\
(\mathbf{n = 7 8 )}\end{array}$ & $\begin{array}{c}\text { Systematic and } \\
\text { targeted biopsy } \\
(\mathbf{n = 1 9 5 )}\end{array}$ & $\begin{array}{c}\text { Mean } \\
\text { difference }\end{array}$ & $\mathbf{9 5 \%}$ CI & p \\
\hline Pain scale & $3.2(2.3)$ & $3.8(2.4)$ & -0.6 & $-1.2,0.0$ & 0.067 \\
\hline Discomfort scale & $3.5(2.5)$ & $4.5(2.6)$ & -1.0 & $-1.7,-0.3$ & 0.005 \\
\hline Anxiety scale & $3.2(2.9)$ & $4.4(3.1)$ & -1.2 & $-2.0,-0.4$ & 0.004 \\
\hline Tolerability scale & $3.2(2.6)$ & $3.4(2.6)$ & -0.2 & $-0.8,0.5$ & 0.7 \\
\hline
\end{tabular}

Scores presented as mean (standard deviation).

\begin{tabular}{|l|c|c|c|c|c|}
\hline \multicolumn{1}{|l|}{ Table 3. Differences in quality of life scales by prior biopsy } \\
\hline & $\begin{array}{c}\text { No prior } \\
\text { biopsy } \\
\mathbf{( n = 8 5 )}\end{array}$ & $\begin{array}{c}\text { Prior biopsy } \\
\mathbf{( n = 1 8 8 )}\end{array}$ & $\begin{array}{c}\text { Mean } \\
\text { difference }\end{array}$ & $\mathbf{9 5 \%}$ CI & p \\
\hline Pain scale & $3.5(2.3)$ & $3.6(2.5)$ & -0.1 & $-0.7,0.5$ & 0.8 \\
\hline Discomfort scale & $4.0(2.3)$ & $4.2(2.7)$ & -0.2 & $-0.9,0.5$ & 0.6 \\
\hline Anxiety scale & $4.4(3.1)$ & $3.9(3.0)$ & 0.5 & $-0.3,1.3$ & 0.2 \\
\hline Tolerability scale & $3.4(2.5)$ & $3.3(2.6)$ & 0.2 & $-0.5,0.8$ & 0.6 \\
\hline
\end{tabular}

Scores are presented as mean (standard deviation). 